\title{
BASIC INVARIANTS OF GEOMETRIC MAPPINGS
}

\author{
NENAD O. VESIĆ
}

Received 18 March, 2019

\begin{abstract}
This study is motivated by the researches in the field for invariants of geodesic and conformal mappings presented in (T. Y. Thomas, [17]) and (H. Weyl, [20]). The Thomas projective parameter and the Weyl projective tensor are generalized in this article. Generators for vector spaces of invariants of geometric mappings are obtained in here.
\end{abstract}

2010 Mathematics Subject Classification: 53A55; 53B05; 53C15

Keywords: invariant of mapping, affine connection, Thomas projective parameter, Weyl projective tensor, curvature tensor, affine connection

\section{INTRODUCTION}

An $N$-dimensional manifold $\mathcal{M}_{N}$ equipped with an affine connection $\nabla$ (with torsion) is called the non-symmetric affine connection space $\mathbb{G A}_{N}$ (see $[6,12-15,18,19$, 21]). As a special case, the manifold $\mathcal{M}_{N}$ equipped with a torsion-free affine connection $\stackrel{0}{\nabla}$ is called the symmetric affine connection space $\mathbb{A}_{N}$. More details about the theory of symmetric affine connection spaces may be found in $[9-11,16]$.

T. Y. Thomas [17] and H. Weyl [20] started the research about invariants of special diffeomorphisms between symmetric affine connection spaces for different applications in physics. Many authors have continued the Thomas's and Weyl's works. J. Mikeš [1, 2, 8-11], I. Hinterleitner [10,11], N. S. Sinyukov [16], are some of them. Some of invariant geometrical object for diffeomorphisms of non-symmetric affine connection spaces are obtained in $[15,18,19,21]$.

In this paper, as in the previous articles, books and monographs, the spaces $\mathbb{G}_{N}$ and $\mathbb{G}_{N}$ will be the manifold $\mathcal{M}_{N}$ equipped with the affine connections $\nabla$ and $\bar{\nabla}=$ $f(\nabla)$. A diffeomorphism $f: \mathbb{G A}_{N} \rightarrow \mathbb{G}_{\mathbb{A}_{N}}$ which the affine connection $\nabla$ of the space $\mathbb{G A}_{N}$ transforms to the affine connection $\bar{\nabla}$ of the space $\mathbb{G}_{\mathbb{A}}$ is the mapping of the space $\mathbb{G A}_{N}$.

The author was supported in part by the Serbian Ministry of Education, Science and Technological Development, Grant No. 174012. 
In this paper, we will obtain sets of families of invariants for diffeomorphisms defined on affine connection spaces with and without torsion. Moreover, we will prove that several of these families of invariants are linearly independent.

\subsection{Affine connection spaces}

For different applications in physics, for example in the Theory of Relativity [3-5], affine connection spaces with torsion have been studied.

Let $\mathbb{G} \mathbb{A}_{N}$ be a non-symmetric affine connection space. The affine connection coefficients $L_{j k}^{i}$ of this space are non-symmetric in the indices $j$ and $k$. The symmetric and anti-symmetric part of the coefficient $L_{j k}^{i}$ are respectively

$$
L_{\underline{j k}}^{i}=\frac{1}{2}\left(L_{j k}^{i}+L_{k j}^{i}\right) \quad \text { and } \quad L_{j k}^{i}=\frac{1}{2}\left(L_{j k}^{i}-L_{k j}^{i}\right) .
$$

The symmetric part $L_{j k}^{i}$ is the affine connection coefficient for a torsion-free affine

connection $\stackrel{0}{\nabla}$. The manifold $\mathcal{M}^{N}$ equipped with the affine connection $\stackrel{0}{\nabla}$ (whose coefficients are $L_{\underline{j k}}^{i}$ ) is the associated space $\mathbb{A}_{N}$ (of the space $\mathbb{G A}_{N}$ ).

The covariant derive of a tensor $a_{j}^{i}$ of the type $(1,1)$ with respect to the affine connection of the associated space $\mathbb{A}_{N}$ is (see $[9-11,16]$ )

$$
a_{j \mid k}^{i}=a_{j, k}^{i}+L_{\underline{\alpha k}}^{i} a_{j}^{\alpha}-L_{\underline{j k}}^{\alpha} a_{\alpha}^{i},
$$

for the partial derivatives $\partial / \partial x^{i}$ denoted by comma.

With respect to the affine connection $\stackrel{0}{\nabla}$ and the corresponding covariant derivative |, one Ricci-type identity is obtained. The corresponding curvature tensor of the associated space $\mathbb{A}_{N}$ is (see $\left.[9-11,16]\right)$

$$
R_{j m n}^{i}=L_{\underline{j m, n}, i}^{i}-L_{\underline{j n, m}}^{i}+L_{\underline{j m}}^{\alpha} L_{\underline{\alpha n}}^{i}-L_{\underline{j n}}^{\alpha} L_{\alpha m}^{i} .
$$

Based on the definitions and results from L. P. Eisenhart [6, 7], A. Einstein [3-5], S. M. Minčić defined four kinds of covariant derivaties with respect to the affine connection $\nabla$ of the space $\mathbb{G A}_{N}[12-14]$

$$
\begin{array}{ll}
a_{j \mid k}^{i}=a_{j, k}^{i}+L_{\alpha k}^{i} a_{j}^{\alpha}-L_{j k}^{\alpha} a_{\alpha}^{i}, & a_{j \mid k}^{i}=a_{j, k}^{i}+L_{k \alpha}^{i} a_{j}^{\alpha}-L_{k j}^{\alpha} a_{\alpha}^{i}, \\
a_{j \mid k}^{i}=a_{j, k}^{i}+L_{\alpha k}^{i} a_{j}^{\alpha}-L_{k j}^{\alpha} a_{\alpha}^{i}, & a_{j \mid k}^{i}=a_{j, k}^{i}+L_{k \alpha}^{i} a_{j}^{\alpha}-L_{j k}^{\alpha} a_{\alpha}^{i} .
\end{array}
$$

With respect to these generalizations of the covariant derivative (1.2), S. M. Minčić got four curvature tensors, eight derived curvature tensors and fifteen curvature pseudotensors of the space $\mathbb{G A}_{N}$ [12-14]. Curvature tensors and derived curvature tensors of the space $\mathbb{G A}_{N}$ are elements of the family [21]

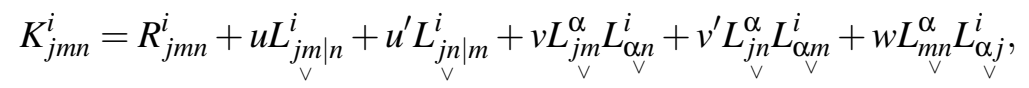


for the curvature tensor $R_{j m n}^{i}$ of the associated space $\mathbb{A}_{N}$ and real coefficients $u, u^{\prime}, v$, $v^{\prime}, w$. Five of twelve curvature tensors from the family (1.5) are linearly independent [13], while the rest can be expressed in terms of these five tensors and the curvature tensor $R_{j m n}^{i}$ of the associated space $\mathbb{A}_{N}$.

Special kind of non-symmetric affine connection spaces are $N$-dimensional differentiable manifolds equipped with the non-symmetric metric tensor $g_{i j}$ of the type $(0,2)$. The symmetric and anti-symmetric part of the metric $g_{i j}$ are

$$
g_{i j}=\frac{1}{2}\left(g_{i j}+g_{j i}\right) \quad \text { and } \quad g_{i j}=\frac{1}{2}\left(g_{i j}-g_{j i}\right) .
$$

These spaces are the generalized Riemannian spaces $\mathbb{G R}_{N}$ (see [7]). The affine connection coefficients of the space $\mathbb{G R}_{N}$ are the generalized Christoffel symbols of the second kind

$$
\Gamma_{j k}^{i}=\frac{1}{2} g^{i \underline{\alpha}}\left(g_{j \alpha, k}-g_{j k, \alpha}+g_{\alpha k, j}\right),
$$

for $[g \underline{i j}]=\left[g_{i j}\right]^{-1}$. After symmetrizing the symbols $\Gamma_{j k}^{i}$ in the indices $j$ and $k$, we get that they reduce to the corresponding Christoffel symbols $\Gamma_{\underline{j k}}^{i}$ obtained from the symmetric metric $g_{i j}$ of the associated space $\mathbb{R}_{N}$.

\section{About InVARIANTS}

Many invariants of mappings for torsion-free spaces are obtained. Some of them are the Thomas projective parameter, the Weyl projective tensor, the Weyl conformal curvature, and many others. These invariants may be found in the next monographs, books and papers: Mikeš [1,2,8-11], Sinyukov [16], Hinterleitner [10], Berezovski $[1,2,10]$, etc.

In this paper, the author's main purpose is to search inceptive invariants of different mappings defined on affine connection spaces. We will start the generalization of the basic invariants in here. The next aim of this article is to discover how many of the basic invariants are linearly independent.

Let $f: \mathbb{G A}_{N} \rightarrow \mathbb{G A}_{N}$ be a mapping between non-symmetric affine connection spaces $\mathbb{G A}_{N}$ and $\mathbb{G}_{N}$.

The deformation tensor $P_{j k}^{i}=\bar{L}_{j k}^{i}-L_{j k}^{i}$ of this mapping is

$$
P_{j k}^{i}=\bar{\omega}_{j k}^{i}-\omega_{j k}^{i}+\bar{\tau}_{j k}^{i}-\tau_{j k}^{i},
$$

for geometrical objects $\omega_{j k}^{i}, \bar{\omega}_{j k}^{i}, \tau_{j k}^{i}, \bar{\tau}_{j k}^{i}$ of the type $(1,2)$ such that $\omega_{j k}^{i}=\omega_{k j}^{i}, \bar{\omega}_{j k}^{i}=$ $\bar{\omega}_{k j}^{i}, \tau_{j k}^{i}=-\tau_{k j}^{i}, \bar{\tau}_{j k}^{i}=-\bar{\tau}_{k j}^{i}$. If the mapping $f$ is equitorsion $[15,19,21]$, i.e. if $\bar{L}_{j k}^{i}=L_{j k}^{i}$, the equation (2.1) reduces to

$$
P_{j k}^{i}=\bar{\omega}_{j k}^{i}-\omega_{j k}^{i}
$$


After symmetrizing the equations $(2.1,2.2)$ in the indices $j$ and $k$, we get

$$
P_{\underline{j k}}^{i}=\bar{\omega}_{j k}^{i}-\omega_{j k}^{i} \text {. }
$$

The deformation tensor $\bar{P}_{j k}^{i}$ of the inverse map $f^{-1}: \mathbb{G}_{\mathbb{A}_{N}} \rightarrow \mathbb{G A}_{N}$ is

$$
\bar{P}_{j k}^{i}=L_{j k}^{i}-\bar{L}_{j k}^{i}=-P_{j k}^{i} .
$$

So, the following equalities hold

$$
P_{\underline{j k}}^{i}=\bar{L}_{\underline{j k}}^{i}-L_{\underline{j k}}^{i}=\bar{\omega}_{j k}^{i}-\omega_{j k}^{i}=\left(-\frac{1}{2} \bar{P}_{\underline{j k}}^{i}\right)-\left(-\frac{1}{2} P_{j k}^{i}\right) .
$$

Hence, the next equation is satisfied

$$
P_{\underline{j k}}^{i}=\bar{\omega}_{(1) \cdot j k}^{i}-\omega_{(1) \cdot j k}^{i}=\bar{\omega}_{(2) \cdot j k}^{i}-\omega_{(2) \cdot j k}^{i}=\bar{\omega}_{(3) \cdot j k}^{i}-\omega_{(3) . j k}^{i},
$$

for the geometrical objects

$$
\omega_{(1) . j k}^{i}=L_{\underline{j k}}^{i}, \quad \omega_{(2) . j k}^{i}=\omega_{j k}^{i}, \quad \omega_{(3) . j k}^{i}=-\frac{1}{2} P_{\underline{j k}}^{i},
$$

and the corresponding $\bar{\omega}_{(1) . j k}^{i}, \bar{\omega}_{(2) . j k}^{i}, \bar{\omega}_{(3) . j k}^{i}$.

Here and after, the symbol $(p)$ means that the equal-index summation convention does not apply to the index $p$.

After anti-symmetrizing the equation (2.1) in the indices $j$ and $k$, we get

$$
P_{j k}^{i}=\xi_{j k}^{i}=\bar{L}_{\substack{j k \\ i}}^{i} L_{j k}^{i}=\bar{\tau}_{j k}^{i}-\tau_{j k}^{i} .
$$

\subsection{Invariants in symmetric affine connection space}

With respect to the equation (2.5), we get

$$
\tilde{\overline{\mathcal{T}}}_{(1) \cdot j k}^{i}=\widetilde{\mathcal{T}}_{(1) \cdot j k}^{i}, \quad \tilde{\overline{\mathcal{T}}}_{(2) . j k}^{i}=\widetilde{\mathcal{T}}_{(2) . j k}^{i}, \quad \widetilde{\overline{\mathcal{T}}}_{(3) \cdot j k}^{i}=\widetilde{\mathcal{T}}_{(3) \cdot j k}^{i},
$$

for the geometrical objects

$$
\widetilde{\mathcal{T}}_{(1) . j k}^{i}=0, \quad \tilde{\mathcal{T}}_{(2) \cdot j k}^{i}=L_{\underline{j k}}^{i}-\omega_{j k}^{i}, \quad \tilde{\mathcal{T}}_{(3) \cdot j k}^{i}=\frac{1}{2}\left(\bar{L}_{\underline{j k}}^{i}+L_{\underline{j k}}^{i}\right),
$$

and the corresponding $\widetilde{\overline{\mathcal{T}}}_{(1) \cdot j k}^{i}, \widetilde{\overline{\mathcal{T}}}_{(2) \cdot j k}^{i}, \widetilde{\overline{\mathcal{T}}}_{(3) \cdot j k}^{i}$.

From the equation

$$
\begin{aligned}
& \tilde{\overline{\mathcal{T}}}_{(p) . j m, n}^{i}-\tilde{\overline{\mathcal{T}}}_{(p) . j n, m}^{i}+\tilde{\overline{\mathcal{T}}}_{(p) . j m}^{\alpha} \tilde{\overline{\mathcal{T}}}_{(p) \cdot \alpha n}^{i}-\tilde{\overline{\mathcal{T}}}_{(p) \cdot j n}^{\alpha} \tilde{\overline{\mathcal{T}}}_{(p) . \alpha m}^{i} \\
& =\widetilde{\mathcal{T}}_{(p) . j m, n}^{i}-\widetilde{\mathcal{T}}_{(p) . j n, m}^{i}+\widetilde{\mathcal{T}}_{(p) . j m}^{\alpha} \tilde{\mathcal{T}}_{(p) . \alpha n}^{i}-\widetilde{\mathcal{T}}_{(p) . j n}^{\alpha} \tilde{\mathcal{T}}_{(p) . \alpha m}^{i},
\end{aligned}
$$

$p=1,2,3$, one obtains that the following equalities are satisfied

for the geometrical objects

$$
\widetilde{\bar{W}}_{(p) . j m n}^{i}=\widetilde{\mathcal{W}}_{(p) . j m n}^{i},
$$

$$
\widetilde{\mathcal{W}}_{(p) . j m n}^{i}=R_{j m n}^{i}-\omega_{(p) \cdot j m \mid n}^{i}+\omega_{(p) \cdot j n \mid m}^{i}+\omega_{(p) . j m}^{\alpha} \omega_{(p) \cdot \alpha n}^{i}-\omega_{(p) \cdot j n}^{\alpha} \omega_{(p) \cdot \alpha m}^{i},
$$


and the corresponding $\widetilde{\widetilde{\mathcal{W}}}_{(p) \text {.jmn }}^{i}$.

Therefore, the following lemma holds.

Lemma 1. Let $f: \mathbb{G A}_{N} \rightarrow \mathbb{G}_{N}$ be a mapping of the associated space $\mathbb{A}_{N}$ characterized by the deformation tensor (2.5).

The geometrical objects $\widetilde{\mathcal{T}}_{(p) . j k}^{i}, p=1,2,3$, given by the equation (2.8) are invariants of the mapping $f$.

The geometrical objects $\widetilde{\mathcal{W}}_{(p) . j m n}^{i}, p=1,2,3$, given by the equation (2.9) are invariants of the mapping $f$.

An invariant $\tilde{\mathcal{T}}_{(p) . j k}^{i}$, for $p=1,2,3$, of the mapping $f: \mathbb{G A}_{N} \rightarrow \mathbb{G}^{\mathbb{A}_{N}}$ is the (basic) $p$-th class associated invariant of the Thomas type. An invariant $\widetilde{\mathcal{W}}_{(p) \cdot j k}^{i}$, for $p=$ $1,2,3$, of the mapping $f: \mathbb{G A}_{N} \rightarrow \mathbb{G}_{\mathbb{A}}$ is the basic p-th class associated invariant of the Weyl type.

Remark 1 . The equalities

$$
\bar{\omega}_{(3) . j m}^{\alpha} \bar{\omega}_{(3) . \alpha n}^{i}=\frac{1}{4} \bar{P}_{\underline{j m}}^{\alpha} \bar{P}_{\underline{\alpha n}}^{i}=\frac{1}{4} P_{\underline{j m}}^{\alpha} P_{\underline{\alpha n}}^{i}=\omega_{(3) . j m}^{\alpha} \omega_{(3) . \alpha n}^{i},
$$

are satisfied. Thus, the invariant $\widetilde{\mathcal{W}}_{(3) . j m n}^{i}$ reduces to

$$
\widetilde{\mathcal{W}}_{(3) \cdot j m n}^{i}=R_{j m n}^{i}-\omega_{(3) . j m \mid n}^{i}+\omega_{(3) \cdot j n \mid m}^{i} .
$$

This invariant is important for researches about invariants of mappings characterized by deformation tensors $P_{\underline{j}}^{i}$ which are not expressed in the form (2.3). The almost geodesic mappings of the first kind are an example of maps such that (see $[1,2,10])$.

Corollary 1. Let in the equation (2.6) be $\omega_{(2) . j k}^{i}=\delta_{j}^{i} \rho_{k}+\delta_{k}^{i} \rho_{j}+\sigma_{j k}^{i}$, for a 1-form $\rho_{j}$ and a geometrical object $\sigma_{j k}^{i}$ of the type $(1,2)$ symmetric in the indices $j$ and $k$.

The geometrical objects

$$
\begin{aligned}
\widetilde{T}_{(2) . j k}^{i}= & \underline{L_{j k}^{i}}-\sigma_{j k}^{i}-\frac{1}{N+1}\left(\left(L_{j \alpha}^{\alpha}-\sigma_{\underline{j \alpha}}^{\alpha}\right) \delta_{k}^{i}+\left(L_{k \alpha}^{\alpha}-\sigma_{\underline{k \alpha}}^{\alpha}\right) \delta_{j}^{i}\right) \\
\widetilde{W}_{(2) . j m n}^{i}= & R_{j m n}^{i}-\sigma_{j m \mid n}^{i}+\sigma_{j n \mid m}^{i}+\sigma_{j m}^{\alpha} \sigma_{\alpha n}^{i}-\sigma_{j n}^{\alpha} \sigma_{\alpha m}^{i} \\
& +\frac{1}{N+1} \delta_{j}^{i}\left(R_{[m n]}+\sigma_{\alpha[m \mid n]}^{\alpha}\right)+\frac{N}{N^{2}-1} \delta_{[m}^{i} R_{j n]}+\frac{1}{N^{2}-1} \delta_{[m}^{i} R_{n] j} \\
& -\frac{1}{N^{2}-1} \delta_{m}^{i}\left(\sigma_{\alpha[j \mid n]}^{\alpha}+(N+1)\left(\sigma_{j n \mid \alpha}^{\alpha}-\sigma_{j \alpha \mid n}^{\alpha}-\sigma_{j n}^{\alpha} \sigma_{\alpha \beta}^{\beta}+\sigma_{j \beta}^{\alpha} \sigma_{n \alpha}^{\beta}\right)\right) \\
& +\frac{1}{N^{2}-1} \delta_{n}^{i}\left(\sigma_{\alpha[j \mid m]}^{\alpha}+(N+1)\left(\sigma_{j m \mid \alpha}^{\alpha}-\sigma_{j \alpha \mid m}^{\alpha}-\sigma_{j m}^{\alpha} \sigma_{\alpha \beta}^{\beta}+\sigma_{j \beta}^{\alpha} \sigma_{\alpha m}^{\beta}\right)\right)
\end{aligned}
$$

are the invariants of the mapping $f$ of the Thomas and the Weyl type respectively. 
Proof. The geometrical objects

$$
\begin{aligned}
& \widetilde{\mathcal{T}}_{(2) . j k}^{i}=L_{j k}^{i}-\delta_{k}^{i} \rho_{j}-\delta_{j}^{i} \rho_{k}-\sigma_{j k}^{i} \\
& \widetilde{\mathcal{W}}_{(2) . j m n}^{i}= R_{j m n}^{i}-\delta_{j}^{i} \rho_{[m \mid n]}-\delta_{m}^{i}\left(\rho_{j \mid n}+\rho_{j} \rho_{n}+\sigma_{j n}^{\alpha} \rho_{\alpha}\right) \\
&+\delta_{n}^{i}\left(\rho_{j \mid m}+\rho_{j} \psi_{m}+\sigma_{j m}^{\alpha} \rho_{\alpha}\right) \\
& \quad-\sigma_{j m \mid n}^{i}+\sigma_{j n \mid m}^{i}+\sigma_{j m}^{\alpha} \sigma_{\alpha n}^{i}-\sigma_{j n}^{\alpha} \sigma_{\alpha m}^{i},
\end{aligned}
$$

are the second class basic associated invariants of the mapping $f$ of the Thomas and the Weyl type.

If we contract the identity $\stackrel{\widetilde{\mathcal{T}}}{(2) . j k}^{i}-\widetilde{\mathcal{T}}_{(2) . j k}^{i}=0$ over $i$ and $k$, we get

$$
(N+1)\left(\bar{\rho}_{j}-\rho_{j}\right)=\bar{L}_{\underline{j \alpha}}^{\alpha}-\bar{\sigma}_{j \alpha}^{\alpha}-L_{\underline{j \alpha}}^{\alpha}+\sigma_{j \alpha}^{\alpha} \text {. }
$$

After substituting the equation (2.15) into the equality $\stackrel{\widetilde{\mathcal{T}}}{(2) . j k}^{i}-\widetilde{\mathcal{T}}_{(2) . j k}^{i}=0$, one confirms that the following equality holds

$$
\widetilde{T}_{(2) . j k}^{i}=\widetilde{T}_{(2) \cdot j k}^{i},
$$

for $\widetilde{T}_{(2) . j k}^{i}$ from the equation (2.11) and the corresponding $\widetilde{T}_{(2) . j k}^{i}$.

Let be $\rho_{i j}=\rho_{j \mid n}+\rho_{j} \psi_{n}+\sigma_{j n}^{\alpha} \rho_{\alpha}$ and $\bar{\rho}_{i j}=\bar{\rho}_{j \mid n}+\bar{\rho}_{j} \bar{\rho}_{n}+\bar{\sigma}_{j n}^{\alpha} \bar{\rho}_{\alpha}$, for the covariant derivative with respect to the affine connection of the torsion-free space $\overline{\mathbb{A}}_{N}$ denoted by $\|$.

With respect to this substitution, the equality $0=\widetilde{\overline{\mathcal{W}}}_{(2) . j m n}^{i}-\widetilde{\mathcal{W}}_{(2) . j m n}^{i}$ transforms to

$$
\begin{aligned}
0= & \bar{R}_{j m n}^{i}-R_{j m n}^{i}-\delta_{j}^{i}\left(\overline{\boldsymbol{\rho}}_{[m n]}-\rho_{[m n]}\right)-\delta_{m}^{i}\left(\overline{\boldsymbol{\rho}}_{j n}-\rho_{j n}\right)+\delta_{n}^{i}\left(\overline{\boldsymbol{\rho}}_{j m}-\rho_{j m}\right) \\
& -\bar{\sigma}_{j m \| n}^{i}+\bar{\sigma}_{j n \| m}^{i}+\bar{\sigma}_{j m}^{\alpha} \bar{\sigma}_{\alpha n}^{i}-\bar{\sigma}_{j n}^{\alpha} \bar{\sigma}_{\alpha m}^{i}+\sigma_{j m \mid n}^{i}-\sigma_{j n \mid m}^{i}-\sigma_{j m}^{\alpha} \sigma_{\alpha n}^{i}+\sigma_{j n}^{\alpha} \sigma_{\alpha m}^{i} .
\end{aligned}
$$

After contracting the equation (2.16) in the indices $i$ and $j$ and using the relations $R_{\alpha m n}^{\alpha}=-\left(R_{m n}-R_{n m}\right) \equiv-R_{[m n]}$, for the alternation in the indices $m$ and $n$ denoted by the square brackets, we get

$$
(N+1)\left(\bar{\rho}_{[m n]}-\rho_{[m n]}\right)=-\bar{R}_{[m n]}+R_{[m n]}-\bar{\sigma}_{\alpha m \| n}^{\alpha}+\bar{\sigma}_{\alpha n \| m}^{\alpha}+\sigma_{\alpha m \mid n}^{\alpha}-\sigma_{\alpha n \mid m}^{\alpha},
$$

i.e.

$$
\begin{aligned}
0= & \bar{R}_{j m n}^{i}+\frac{1}{N+1} \delta_{j}^{i}\left(\bar{R}_{[m n]}+\bar{\sigma}_{\alpha[m \mid n]}^{\alpha}\right)+\delta_{n}^{i}\left(\bar{\rho}_{j m}-\rho_{j m}\right)-\delta_{m}^{i}\left(\bar{\rho}_{j n}-\rho_{j n}\right) \\
& -\bar{\sigma}_{j m \mid n}^{i}+\bar{\sigma}_{j n \| m}^{i}+\bar{\sigma}_{j m}^{\alpha} \bar{\sigma}_{\alpha n}^{i}-\bar{\sigma}_{j n}^{\alpha} \bar{\sigma}_{\alpha m}^{i}-R_{j m n}^{i}-\frac{1}{N+1} \delta_{j}^{i}\left(R_{[m n]}+\sigma_{\alpha[m \mid n]}^{\alpha}\right) \\
& +\sigma_{j m \mid n}^{i}-\sigma_{j n \mid m}^{i}-\sigma_{j m}^{\alpha} \sigma_{\alpha n}^{i}+\sigma_{j n}^{\alpha} \sigma_{\alpha m}^{i} .
\end{aligned}
$$


If we contract the last equation over $i$ and $n$, we obtain that the following equation is satisfied

$$
\begin{aligned}
(N-1)\left(\bar{\rho}_{j m}-\rho_{j m}\right)= & -\bar{R}_{j m}+\frac{1}{N+1}\left(\bar{R}_{[j m]}+\bar{\sigma}_{\alpha[j \| m]}^{\alpha}\right) \\
& +R_{j m}-\frac{1}{N+1}\left(R_{[j m]}+\sigma_{\alpha[j \mid m]}^{\alpha}\right) \\
& +\bar{\sigma}_{j m \mid \alpha}^{\alpha}-\bar{\sigma}_{j \alpha \| m}^{\alpha}-\bar{\sigma}_{j m}^{\alpha} \bar{\sigma}_{\alpha \beta}^{\beta}+\bar{\sigma}_{j \beta}^{\alpha} \bar{\sigma}_{\alpha m}^{\beta} \\
& -\sigma_{j m \mid \alpha}^{\alpha}+\sigma_{j \alpha \mid m}^{\alpha}+\sigma_{j m}^{\alpha} \sigma_{\alpha \beta}^{\beta}-\sigma_{j \beta}^{\alpha} \sigma_{\alpha m}^{\beta} .
\end{aligned}
$$

Based on the equations $(2.17,2.18)$, one gets

$$
\widetilde{\bar{W}}_{(2) \cdot j m n}^{i}=\widetilde{W}_{(2) \cdot j m n}^{i},
$$

for the geometrical object $\widetilde{W}_{(2) . j m n}^{i}$ from the equation (2.12) and the corresponding $\widetilde{\bar{W}}_{(2) . j m n}^{i}$.

The invariants $(2.11,2.12)$ are the (second kind) derived associated invariants of the mapping $f$ of the Thomas and Weyl type, respectively.

Remark 2. If the deformation tensor $P_{j k}^{i}$ of a studied mapping is expressed in the form (2.1) all invariants of the Weyl type reduce to the corresponding invariants of the second class. If the deformation tensor $P_{j k}^{i}$ of a mapping satisfies a differential equation, it may be obtained just the Weyl type invariants of the third class. The invariants of the first and the second class of the Thomas type produce the multiplied families of invariants for mappings. Hence, it is enough to obtain invariants $\mathcal{W}_{(p) \text {.jmn }}^{i}$ for one $p$. All other invariants $\widetilde{\mathcal{W}}$ of the Weyl type reduce to the obtained one.

\subsection{Invariants in non-symmetric affine connection space}

We will generalize the invariants $(2.8,2.9)$ in this part of the paper.

With respect to the equation (2.7), one gets

$$
\hat{\overline{\mathcal{T}}}^{i}{ }^{i}=\hat{\mathcal{T}}^{i}{ }_{j k},
$$

for the geometrical object

$$
\hat{\mathcal{T}}^{i}{ }_{j k}=L_{j k}^{i}-\tau_{j k}^{i}
$$

and the corresponding $\hat{\overline{\mathcal{T}}}_{j k}^{i}$. Based on the equations $(2.8,2.19)$, one obtains that it is satisfied the equalities

$$
\overline{\mathcal{T}}_{(1) . j k}^{i}=\mathcal{T}_{(1) . j k}^{i}, \quad \overline{\mathcal{T}}_{(2) . j k}^{i}=\mathcal{T}_{(2) . j k}^{i}, \quad \overline{\mathcal{T}}_{(3) . j k}^{i}=\mathcal{T}_{(3) . j k}^{i},
$$

for the geometrical objects

$$
\mathcal{T}_{(1) . j k}^{i}=L_{j k}^{i}-\tau_{j k}^{i}, \quad \mathcal{T}_{(2) . j k}^{i}=L_{j k}^{i}-\omega_{j k}^{i}-\tau_{j k}^{i}, \quad \mathcal{T}_{(3) . j k}^{i}=L_{j k}^{i}+\frac{1}{2} P_{\underline{~ j k}}^{i}-\tau_{j k}^{i},
$$


and the corresponding $\overline{\mathcal{T}}_{(1) \cdot j k}^{i}, \overline{\mathcal{T}}_{(2) . j k}^{i}, \overline{\mathcal{T}}_{(3) \cdot j k}^{i}$.

Lemma 2. Let $f: \mathbb{G A}_{N} \rightarrow \mathbb{G}_{N}$ be a mapping of the non-symmetric affine connection space $\mathbb{G A}_{N}$. The geometrical objects $\hat{\mathcal{T}}^{i}{ }_{j k}$ and $\mathcal{T}_{(p) . j k}^{i}$, respectively given by the equations $(2.19,2.20)$, are invariants of the mapping $f$.

Corollary 2. The invariants $(2.8,2.19,2.20)$ satisfy the equation

$$
\mathcal{T}_{(p) . j k}^{i}=\widetilde{\mathcal{T}}_{(p) . j k}^{i}+\hat{\mathcal{T}}^{i}{ }_{j k},
$$

for $p=1,2,3$.

The invariant $\mathcal{T}_{(p) . j k}^{i}$, for $p=1,2,3$, is the $p$-th class general invariant of the Thomas type. The invariant $\hat{\mathcal{T}}_{j k}^{i}$ is the anti-symmetric invariant of the Thomas type.

From the difference $\hat{\mathcal{T}}^{i}{ }_{j m \mid n}-\hat{\mathcal{T}}_{j m \mid n}^{i}$ and the equality $\hat{\mathcal{T}}_{j m}^{\alpha} \hat{\overline{\mathcal{T}}}_{\alpha n}^{i}-\hat{\mathcal{T}}_{j m}^{\alpha} \hat{\mathcal{T}}_{\alpha n}^{i}=0$, we obtain the following transformation rules

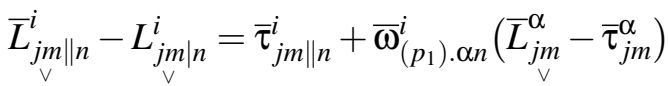

$$
\begin{aligned}
& -\bar{\omega}_{\left(p_{2}\right) . j n}^{\alpha}\left(\bar{L}_{\alpha m}^{i}-\bar{\tau}_{\alpha m}^{i}\right)-\bar{\omega}_{\left(p_{3}\right) . m n}^{\alpha}\left(\bar{L}_{j \alpha}^{i}-\bar{\tau}_{j \alpha}^{i}\right) \\
& -\tau_{j m \mid n}^{i}-\omega_{\left(p_{1}\right) . \alpha n}^{i}\left(L_{j m}^{\alpha}-\tau_{j m}^{\alpha}\right) \\
& +\omega_{\left(p_{2}\right) . j n}^{\alpha}\left(L_{\alpha \mathrm{v}}^{i}-\tau_{\alpha m}^{i}\right)+\omega_{\left(p_{3}\right) . m n}^{\alpha}\left(L_{j \alpha}^{i}-\tau_{j \alpha}^{i}\right),
\end{aligned}
$$

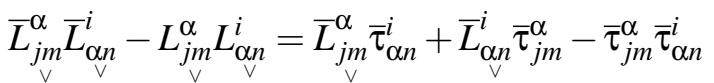

$$
\begin{aligned}
& -L_{j m}^{\alpha} \tau_{\alpha n}^{i}-L_{\alpha n}^{i} \tau_{j m}^{\alpha}+\tau_{j m}^{\alpha} \tau_{\alpha n}^{i},
\end{aligned}
$$

for $p_{1}, p_{2}, p_{3}=1,2$.

Remark 3. The following equalities are satisfied

$$
\begin{aligned}
\bar{\omega}_{(3) . j k}^{i} \hat{\overline{\mathcal{T}}}_{m n}^{l}-\omega_{(3) . j k}^{i} \hat{\mathcal{T}}_{m n}^{l} & =-\frac{1}{2} \overline{\mathcal{P}}_{j k}^{i} \hat{\overline{\mathcal{T}}}_{m n}^{l}+\frac{1}{2} P_{\underline{j k}}^{i} \hat{\mathcal{T}}_{m n}^{l} \\
& =\bar{\omega}_{(1) . j k}^{i} \hat{\overline{\mathcal{T}}}_{m n}^{l}-\omega_{(1) . j k}^{i} \hat{\mathcal{T}}_{m n}^{l} .
\end{aligned}
$$

Hence, the geometrical objects $\omega_{(1) . j k}^{i}, \omega_{(2) . j k}^{i}$, are enough to express all transformation rules (2.22) with respect to a mapping $f: \mathbb{G A}_{N} \rightarrow \mathbb{G}^{\mathbb{A}} \bar{A}_{N}$.

Let us currently express the invariants (2.9) in the form

$$
\widetilde{\mathcal{W}}_{(p) . j m n}^{i}=R_{j m n}^{i}-\widetilde{\mathcal{D}}_{(p) \cdot j m n}^{i},
$$


for the corresponding geometrical objects $\widetilde{\mathcal{D}}_{(p) . j m n}^{i}$. From this expression, and the equalities $\widetilde{\widetilde{W}}_{(p) . j m n}^{i}-\widetilde{\mathcal{W}}_{(p) . j m n}^{i}=0, p=1,2,3$, we get

$$
\bar{R}_{j m n}^{i}-R_{j m n}^{i}=\widetilde{\bar{D}}_{(p) . j m n}^{i}-\widetilde{\mathcal{D}}_{(p) . j m n}^{i} .
$$

With respect to the equation (1.5), we obtain that the following equation holds

$$
\begin{aligned}
& \bar{K}_{j m n}^{i}-K_{j m n}^{i}=\bar{R}_{j m n}^{i}-R_{j m n}^{i}+u\left(\bar{L}_{j \downarrow \| n}^{i}-L_{j m \mid n}^{i}\right)
\end{aligned}
$$

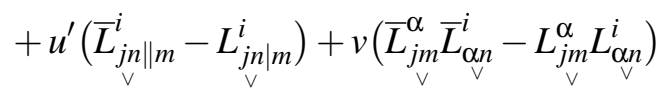

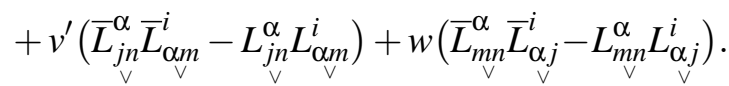

After substituting the results $(2.22,2.23,2.24)$ into the equation $(2.25)$, we get

$$
\overline{\mathcal{W}}_{(p) \cdot\left(p^{1}\right) \cdot\left(p^{2}\right) \cdot j m n}^{i}=\mathcal{W}_{(p) \cdot\left(p^{1}\right) \cdot\left(p^{2}\right) \cdot j m n}^{i},
$$

for $p=1,2,3, p_{1}^{1}, \ldots, p_{3}^{2}=1,2$, the family geometrical objects

$$
\begin{aligned}
\mathcal{W}_{(p) \cdot\left(p^{1}\right) \cdot\left(p^{2}\right) \cdot j m n}^{i}= & K_{j m n}^{i}-\omega_{(p) \cdot j m \mid n}^{i}+\omega_{(p) \cdot j n \mid m}^{i}+\omega_{(p) \cdot j m}^{\alpha} \omega_{(p) . \alpha n}^{i}-\omega_{(p) \cdot j n}^{\alpha} \omega_{(p) \cdot \alpha m}^{i} \\
& -u\left(\tau_{j m \mid n}^{i}+\omega_{\left(p_{1}^{1}\right) \cdot \alpha n}^{i}\left(L_{j m}^{\alpha}-\tau_{j m}^{\alpha}\right)-\omega_{\left(p_{2}^{1}\right) \cdot j n}^{\alpha}\left(L_{\alpha m}^{i}-\tau_{\alpha m}^{i}\right)\right) \\
& -u^{\prime}\left(\tau_{j n \mid m}^{i}+\omega_{\left(p_{1}^{2}\right) \cdot \alpha m}^{i}\left(L_{j n}^{\alpha}-\tau_{j n}^{\alpha}\right)-\omega_{\left(p_{2}^{2}\right) \cdot j m}^{\alpha}\left(L_{\alpha,}^{i}-\tau_{\alpha n}^{i}\right)\right) \\
& +\left(u \omega_{\left(p_{3}^{1}\right) . m n}^{\alpha}+u^{\prime} \omega_{\left(p_{3}^{2}\right) \cdot m n}^{\alpha}\right)\left(L_{j \alpha}^{i}-\tau_{j \alpha}^{i}\right),
\end{aligned}
$$

the real coefficients $u, u^{\prime}, v, v^{\prime}, w$ and the corresponding $\widetilde{\widetilde{\mathcal{W}}}_{(p) \cdot\left(p^{1}\right) \cdot\left(p^{2}\right) . j m n}$.

The following theorem holds.

Theorem 1. Let $f: \mathbb{G} \mathbb{A}_{N} \rightarrow \mathbb{G}^{-} \overline{\mathbb{A}}_{N}$ be a mapping of the non-symmetric affine connection space $\mathbb{G A}_{N}$. The set $\mathcal{W}_{(p) \cdot\left(p^{1}\right) .\left(p^{2}\right) . j m n}^{i}, p=1,2,3, p^{k}=\left(p_{1}^{k}, p_{2}^{k}, p_{3}^{k}\right)$, $k=1,2, p_{r}^{k}=1,2$, of families of geometrical objects given in the equation (2.26) is the set of families of invariants of the Weyl type for the mapping $f$. 
Corollary 3. The invariants $(2.9,2.26)$ of the mapping $f$ satisfy the equation

$$
\begin{aligned}
& \mathcal{W}_{(p) \cdot\left(p^{1}\right) \cdot\left(p^{2}\right) \cdot j m n}^{i}=\widetilde{\mathcal{W}}_{(p) \cdot j m n}^{i} \\
& +u\left(L_{j m \mid n}^{i}-\tau_{j m \mid n}^{i}-\omega_{\left(p_{1}^{1}\right) . \alpha n}^{i}\left(L_{j m}^{\alpha}-\tau_{j m}^{\alpha}\right)+\omega_{\left(p_{2}^{1}\right) . j n}^{\alpha}\left(L_{\alpha m}^{i}-\tau_{\alpha m}^{i}\right)\right) \\
& +u^{\prime}\left(L_{j n \mid m}^{i}-\tau_{j n \mid m}^{i}-\omega_{\left(p_{1}^{2}\right) \cdot \alpha m}^{i}\left(L_{j n}^{\alpha}-\tau_{j n}^{\alpha}\right)-\omega_{\left(p_{2}^{2}\right) \cdot j m}^{\alpha}\left(L_{\alpha n}^{i}-\tau_{\alpha n}^{i}\right)\right) \\
& +\left(u \omega_{\left(p_{3}^{1}\right) \cdot m n}^{\alpha}+u^{\prime} \omega_{\left(p_{3}^{2}\right) . m n}^{\alpha}\right)\left(L_{j \alpha}^{i}-\tau_{j \alpha}^{i}\right)+v\left(L_{j m}^{\alpha}-\tau_{j m}^{\alpha}\right)\left(L_{\alpha,}^{i}-\tau_{\alpha n}^{i}\right) \\
& +v^{\prime}\left(L_{j n}^{\alpha}-\tau_{j n}^{\alpha}\right)\left(L_{\alpha m}^{i}-\tau_{\alpha m}^{i}\right)+w\left(L_{m n}^{\alpha}-\tau_{m n}^{\alpha}\right)\left(L_{\alpha j}^{i}-\tau_{\alpha j}^{i}\right),
\end{aligned}
$$

for $p=1,2,3, p_{1}^{1}, \ldots, p_{3}^{2}=1,2$.

Corollary 4. Let $f: \mathbb{G A}_{N} \rightarrow \mathbb{G}_{\mathbb{A}_{N}}$ be an equitorsion mapping between non-symmetric affine connection spaces $\mathbb{G A}_{N}$ and $\mathbb{G}_{N}$. The invariants $(2.20)$ of this mapping reduce to the corresponding invariants (2.8). The invariant (2.19) coincides with the anti-symmetric part $L_{j k}^{i}$ of the affine connection coefficient $L_{j k}^{i}$.

The set (2.26) of families of invariants of the mapping $f$ reduces to

$$
\begin{aligned}
& \mathcal{W}_{(p) \cdot\left(p^{1}\right) \cdot\left(p^{2}\right) . j m n}^{i}=K_{j m n}^{i}-\omega_{(p) . j m \mid n}^{i}+\omega_{(p) . j n \mid m}^{i}+\omega_{(p) . j m}^{\alpha} \omega_{(p) . \alpha n}^{i}-\omega_{(p) . j n}^{\alpha} \omega_{(p) . \alpha m}^{i}
\end{aligned}
$$

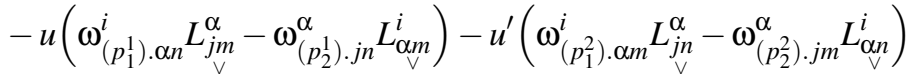

$$
\begin{aligned}
& +\left(u \omega_{\left(p_{3}^{1}\right) \cdot m n}^{\alpha}+u^{\prime} \omega_{\left(p_{3}^{2}\right) \cdot m n}^{\alpha}\right) L_{j \alpha}^{i},
\end{aligned}
$$

for $p=1,2,3, p_{1}^{1}, \ldots, p_{3}^{2}=1,2$.

The equation (2.27) reduces to

$$
\begin{aligned}
& \mathcal{W}_{(p) \cdot\left(p^{1}\right) \cdot\left(p^{2}\right) . j m n}^{i}=\widetilde{\mathcal{W}}_{(p) \cdot j m n}^{i}+\left(u \omega_{\left(p_{3}^{1}\right) \cdot m n}^{\alpha}+u^{\prime} \omega_{\left(p_{3}^{2}\right) \cdot m n}^{\alpha}\right) L_{j \alpha}^{i} \\
& +u\left(L_{j m \mid n}^{i}-\omega_{\left(p_{1}^{1}\right) \cdot \alpha n}^{i} L_{j m}^{\alpha}+\omega_{\left(p_{2}^{1}\right) . j n}^{\alpha} L_{\vee}^{i}\right) \\
& +u^{\prime}\left(L_{j n \mid m}^{i}-\omega_{\left(p_{1}^{2}\right) \cdot \alpha m}^{i} L_{j n}^{\alpha}-\omega_{\left(p_{2}^{2}\right) \cdot j m}^{\alpha} L_{\vee}^{i}\right) \\
& +v L_{j m}^{\alpha} L_{\alpha_{\vee}}^{i}+v^{\prime} L_{j n}^{\alpha} L_{\alpha m}^{i}+w L_{m_{v}}^{\alpha} L_{\alpha j}^{i}
\end{aligned}
$$

for $p=1,2,3, p_{1}^{1}, \ldots, p_{3}^{2}=1,2$.

The sets of invariants $\mathcal{W}_{(p) .\left(p^{1}\right) .\left(p^{2}\right) . j m n}^{i}$, given by the equations $(2.26,2.28)$, are the sets of the $\left(p \cdot p^{1} \cdot p^{2}\right)$-th class invariants of the Weyl type.

The families of invariants in the set (2.26), and in the set (2.28) as well, are equivalent invariants of the mapping $f: \mathbb{G A}_{N} \rightarrow \mathbb{G}_{\mathbb{A}_{N}}$. Because these families are different in general, we are aimed to find how many of these families are linearly independent. 
The set $\mathcal{W}_{(p) .\left(p^{1}\right) \cdot\left(p^{2}\right) . j m n}^{i}$ of invariants may be expressed as

$$
\begin{aligned}
& \mathcal{W}_{(p) \cdot\left(p^{1}\right) \cdot\left(p^{2}\right) \cdot j m n}^{i} \\
& =\widetilde{\mathcal{W}}_{(p) . j m n}^{i}+u \hat{\mathcal{T}}_{j m \mid n}^{i}+u^{\prime} \hat{\mathcal{T}}^{i}{ }_{j n \mid m}+v \hat{\mathcal{T}}_{j m}^{\alpha} \hat{\mathcal{T}}_{\alpha n}^{i}+v^{\prime} \hat{\mathcal{T}}_{j n}^{\alpha} \hat{\mathcal{T}}_{\alpha m}^{i}+w \hat{\mathcal{T}}_{m n}^{\alpha} \hat{\mathcal{T}}_{\alpha j}^{i} \\
& -c_{p}^{1} u L_{\underline{\alpha n}}^{i} \hat{\mathcal{T}}_{j m}^{\alpha}-c_{p}^{2} u \omega_{(p) . \alpha n}^{i} \hat{\mathcal{T}}_{j m}^{\alpha}+c_{p}^{3} u L_{\underline{j n}}^{\alpha} \hat{\mathcal{T}}_{\alpha m}^{i}+c_{p}^{4} u \omega_{(p) . j n}^{\alpha} \hat{\mathcal{T}}_{\alpha m}^{i} \\
& -c_{p}^{7} u^{\prime} L_{\alpha m}^{i} \hat{\mathcal{T}}_{j n}^{\alpha}-c_{p}^{8} u^{\prime} \omega_{(p) . \alpha m}^{i} \hat{\mathcal{T}}_{j n}^{\alpha}+c_{p}^{9} u^{\prime} L_{j m}^{\alpha} \hat{\mathcal{T}}_{\alpha n}^{i}+c_{p}^{10} u^{\prime} \omega_{(p) . j m}^{\alpha} \hat{\mathcal{T}}_{\alpha n}^{i} \\
& +\left(c_{p}^{5} u+c_{p}^{11} u^{\prime}\right) L_{\underline{m n}}^{\alpha} \hat{\mathcal{T}}_{j \alpha}^{i}+\left(c_{p}^{6} u+c_{p}^{12} u^{\prime}\right) \omega_{(p) . m n} \hat{\mathcal{T}}_{j \alpha}^{i},
\end{aligned}
$$

for the corresponding coefficients $c_{p}^{k} \in\{0,1\}, k=1, \ldots, 12$.

There are 64 families of invariants in the set (2.30) characterized by the corresponding 11-tuples

$$
\begin{gathered}
c_{k}=\left(1,-c_{p k}^{1} u,-c_{p k}^{2} u, c_{p k}^{3} u, c_{p k}^{4} u,-c_{p k}^{7} u^{\prime},-c_{p k}^{8} u^{\prime},\right. \\
\left.c_{p k}^{9} u^{\prime}, c_{p k}^{10} u^{\prime}, c_{p k}^{5} u+c_{p k}^{11} u^{\prime}, c_{p k}^{6} u+c_{p k}^{12} u^{\prime}\right),
\end{gathered}
$$

$k=1, \ldots, 64$, for $c_{p k}^{r}=c_{p}^{r}$ in the $k$-th of families of invariants in the set $\widetilde{\mathcal{W}}_{(p) \cdot\left(p^{1}\right) \cdot\left(p^{2}\right) \cdot j m n}$.

The rank of the matrix $\left[\begin{array}{c}c_{1} \\ \vdots \\ c_{64}\end{array}\right]$ of the type $64 \times 13$ is 6 .

The following theorem holds.

Theorem 2. The set (2.26) of invariants of a mapping $f: \mathbb{G A}_{N} \rightarrow \mathbb{G}_{N}$, generates the 6-dimensional vector space.

\section{APPLiCATIONS AND EXAMPLES}

From the above obtained results, we will search invariants of equitorsion geodesic mappings defined on a generalized Riemannian space $\mathbb{G R}_{N}$. Furthermore, we will obtain an associated basic invariant of Weyl type of an almost geodesic mapping $[1,2,10]$ defined on a Riemannian space $\mathbb{R}_{N}$. These invariants will be applied in the examples after theoretical researches.

Equitorsion geodesic mappings. Let $f: \mathbb{G} \mathbb{R}_{N} \rightarrow \mathbb{G} \overline{\mathbb{R}}_{N}$ be an equitorsion geodesic mapping. This mapping is characterized by the following equation

$$
P_{j k}^{i}=\psi_{j} \delta_{k}^{i}+\psi_{k} \delta_{j}^{i}
$$

for the deformation tensor $P_{j k}^{i}=\bar{L}_{j k}^{i}-L_{j k}^{i}$ and a 1-form $\psi_{j}$.

After symmetrizing this equation in the indices $j$ and $k$, we get

$$
P_{\underline{j k}}^{i}=\psi_{j} \delta_{k}^{i}+\psi_{k} \delta_{j}^{i}
$$


If we contract the last equation over $i$ and $k$, we will obtain the following expression of the 1 -form $\psi_{j}$

$$
\psi_{j}=\frac{1}{N+1}\left(\bar{\Gamma}_{\underline{j \alpha}}^{\alpha}-\Gamma_{\underline{j \alpha}}^{\alpha}\right) .
$$

After substituting this expression into the equation (3.2) and recalling the equation (2.3), we get

$$
\omega_{(2) \cdot j k}^{i}=\frac{1}{N+1} \delta_{j}^{i} \Gamma_{\underline{k \alpha}}^{\alpha}+\frac{1}{N+1} \delta_{k}^{i} \Gamma_{\underline{j \alpha}}^{\alpha} .
$$

If we substitute this expression into the equations $(2.8,2.9)$, we obtain that the geometrical objects

$$
\begin{aligned}
\widetilde{\mathcal{T}}_{(2) . j k}^{i}= & \Gamma_{\underline{j k}}^{i}-\frac{1}{N+1}\left(\delta_{j}^{i} \Gamma_{\underline{k \alpha}}^{\alpha}+\delta_{k}^{i} \Gamma_{\underline{j \alpha}}^{\alpha}\right), \\
\widetilde{\mathcal{W}}_{(2) . j m n}^{i}= & R_{j m n}^{i}-\frac{1}{(N+1)^{2}} \delta_{m}^{i}\left((N+1) \Gamma_{\underline{j \alpha \mid n}}^{\alpha}+\Gamma_{\underline{j \alpha}}^{\alpha} \Gamma_{n \underline{n \alpha}}^{\alpha}\right) \\
& \quad+\frac{1}{(N+1)^{2}} \delta_{n}^{i}\left((N+1) \Gamma_{\underline{j \alpha \mid m}}^{\alpha}+\Gamma_{\underline{j \alpha}}^{\alpha} \Gamma_{\underline{m \alpha}}^{\alpha}\right),
\end{aligned}
$$

are the basic associated invariants of the Thomas and the Weyl type of the mapping $f$.

Example 1. Let $\mathbb{G} \mathbb{R}_{3}$ be a generalized Riemannian space equipped with the nonsymmetric metric

$$
g_{i j}=\left[\begin{array}{ccc}
\left(x^{1}\right)^{2} & x^{1} & x^{2} \\
-x^{1} & \left(x^{2}\right)^{2} & x^{3} \\
-x^{2} & -x^{3} & \left(x^{3}\right)^{2}
\end{array}\right]
$$

The symmetric and anti-symmetric part of this metric are

$$
g_{\underline{i j}}=\left[\begin{array}{ccc}
\left(x^{1}\right)^{2} & 0 & 0 \\
0 & \left(x^{2}\right)^{2} & 0 \\
0 & 0 & \left(x^{3}\right)^{2}
\end{array}\right] \text { and } g_{i j}=\left[\begin{array}{ccc}
0 & x^{1} & x^{2} \\
-x^{1} & 0 & x^{3} \\
-x^{2} & -x^{3} & 0
\end{array}\right]
$$

The contravariant metric tensor $\left[g^{i \underline{j}}\right]=\left[g_{i \underline{j}}\right]^{-1}$ of the space $\mathbb{G R}_{3}$ is

$$
g^{i j}=\left[\begin{array}{ccc}
\left(x^{1}\right)^{-2} & 0 & 0 \\
0 & \left(x^{2}\right)^{-2} & 0 \\
0 & 0 & \left(x^{3}\right)^{-2}
\end{array}\right] .
$$

To obtain the invariants (2.29), we need to search the corresponding invariant (2.9), the geometrical objects $\omega_{(1) . j k}^{i}$ and $\omega_{(2) . j k}^{i}$ and the anti-symmetric part $\Gamma_{j k}^{i}$ of the generalized Christoffel symbol $\Gamma_{j k}^{i}$ given by the equation (1.7). 
The affine connection coefficients of the associated space $\mathbb{R}_{3}$ are the Chrisfoffel symbols

$$
\Gamma_{\underline{j k}}^{i}=\omega_{(1) \cdot j k}^{i}=\left\{\begin{array}{cl}
\left(x^{i}\right)^{-1}, & i=j=k \\
0, & \text { otherwise. }
\end{array}\right.
$$

Furthermore, based on the equation (3.3) we get

$$
\omega_{(2) \cdot j k}^{i}=\frac{1}{N+1}\left(\delta_{j}^{i}\left(x^{k}\right)^{-1}+\delta_{k}^{i}\left(x^{j}\right)^{-1}\right) .
$$

For this reason, the curvature tensor of the associated space $\mathbb{R}_{3}$ is $R_{j m n}^{i}=0$. Moreover, the associated invariant of Weyl type (3.5) is

$$
\begin{aligned}
\widetilde{\mathcal{W}}_{(2) . j m n}^{i}= & -\frac{1}{(N+1)^{2}} \delta_{m}^{i}\left((N+1)\left(\left(x^{j}\right)^{-1}\right)_{\mid n}+\left(x^{j}\right)^{-1}\left(x^{n}\right)^{-1}\right) \\
& +\frac{1}{(N+1)^{2}} \delta_{n}^{i}\left((N+1)\left(\left(x^{j}\right)^{-1}\right)_{\mid m}+\left(x^{j}\right)^{-1}\left(x^{m}\right)^{-1}\right) .
\end{aligned}
$$

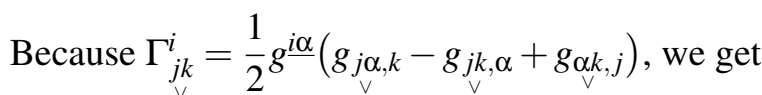

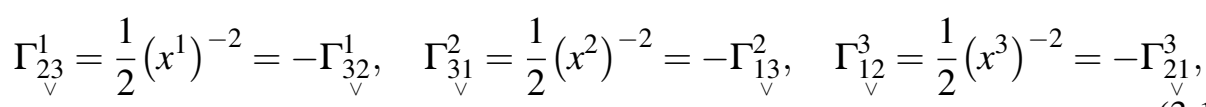

and $\Gamma_{j k}^{i}=0$ in all other cases.

After substituting the expression (3.9) into the equation (2.29) and with respect to the equations $(3.10-3.12)$, for $p_{1}^{1}, \ldots, p_{3}^{2} \in\{1,2\}$, we get that the set of invariants of Weyl type of the mapping $f$ is

$$
\begin{aligned}
& \widetilde{\mathcal{W}}_{(2) \cdot\left(p^{1}\right) \cdot\left(p^{2}\right) \cdot j m n}^{i}=-\frac{1}{(N+1)^{2}} \delta_{m}^{i}\left((N+1)\left(\left(x^{j}\right)^{-1}\right)_{\mid n}+\left(x^{j}\right)^{-1}\left(x^{n}\right)^{-1}\right) \\
& +\frac{1}{(N+1)^{2}} \delta_{n}^{i}\left((N+1)\left(\left(x^{j}\right)^{-1}\right)_{\mid m}+\left(x^{j}\right)^{-1}\left(x^{m}\right)^{-1}\right) \\
& +\left(u \omega_{\left(p_{3}^{1}\right) \cdot m n}^{\alpha}+u^{\prime} \omega_{\left(p_{3}^{2}\right) \cdot m n}^{\alpha}\right) \\
& +u\left(\Gamma_{j m \mid n}^{i}-\omega_{\left(p_{1}^{1}\right) . \alpha n}^{i} \Gamma_{j m}^{\alpha}+\omega_{\left(p_{2}^{1}\right) \cdot j n}^{\alpha} \Gamma_{\vee}^{i}\right) \\
& +u^{\prime}\left(\Gamma_{j \vee}^{i} \mid m-\omega_{\left(p_{1}^{2}\right) \cdot \alpha m}^{i} \Gamma_{\mathrm{v} n}^{\alpha}-\omega_{\left(p_{2}^{2}\right) \cdot j m}^{\alpha} \Gamma_{\mathrm{V} n}^{i}\right)
\end{aligned}
$$

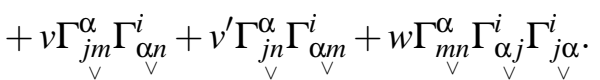

Almost geodesic mappings. N. S. Sinyukov [16], J. Mikeš [1, 2, 8, 10] and many other authors have developed the concept of geodesics. We will search an associated basic invariant of the mapping $f: \mathbb{R}_{N} \rightarrow \overline{\mathbb{R}}_{N}$ characterized by the equation

$$
P_{\underline{n m \mid j}}^{i}+P_{\underline{j m} \mid n}^{i}+P_{\underline{j m}}^{\alpha} P_{\underline{\alpha n}}^{i}+P_{\underline{n m}}^{\alpha} \underline{P_{j}}{ }^{i}=\delta_{j}^{i} a_{m n}+\delta_{n}^{i} a_{m j},
$$


for the tensor $a_{i j}$ of the type $(0,2)$ symmetric by the indices $i$ and $j$. This mapping is called the almost geodesic mapping of the type $\tilde{\pi}_{1}$.

After replacing the indices $m \leftrightarrow n$, we get

$$
P_{\underline{j n} \mid m}^{i}+P_{\underline{m n} \mid j}^{i}+P_{\underline{j n}}^{\alpha} P_{\underline{\alpha m}}^{i}+P_{\underline{m n}}^{\alpha} P_{\underline{\alpha j}}^{i}=\delta_{j}^{i} a_{n m}+\delta_{m}^{i} a_{n j} .
$$

If we subtract the equations (3.13) and (3.14), we will obtain that the following equation is satisfied

$$
P_{\underline{j m \mid n}}^{i}-P_{\underline{j n} \mid m}^{i}=-P_{\underline{j m}}^{\alpha} P_{\underline{\alpha n}}^{i}+P_{\underline{j n}}^{\alpha} P_{\underline{\alpha m}}^{i}+\delta_{n}^{i} a_{j m}-\delta_{m}^{i} a_{j n} .
$$

Based on the equations $(2.10,3.15)$ and the invariance $\bar{P}_{\underline{j m}}^{\alpha} \bar{P}_{\alpha \underline{n}}^{i}=$ $P_{\underline{j m}}^{\alpha} P_{\underline{\alpha n}}^{i}$, we obtain that the geometrical object

$$
\widetilde{\mathcal{W}}_{(3) \cdot j m n}^{i}=R_{j m n}^{i}+\frac{1}{2} \delta_{m}^{i} a_{j n}-\frac{1}{2} \delta_{n}^{i} a_{j m},
$$

is the associated basic invariant of the Weyl type of the mapping $f$.

After contracting the equality $\widetilde{\widetilde{\mathcal{W}^{\prime}}} i$.jmn $-\widetilde{\mathcal{W}}_{(3) \text {.jmn }}^{i}=0$ by the indices $i$ and $n$, we obtain that the curvature tensor $R_{j m n}^{i}$ is the derived invariant of this mapping.

Example 2. Let $\mathbb{R}_{3}$ be a Riemannian space equipped with the symmetric metric $g_{i j}$ given in the equation (3.7). Let also $f: \mathbb{R}_{3} \rightarrow \overline{\mathbb{R}}_{3}$ be an almost geodesic mapping of the type $\tilde{\pi}_{1}$.

As we obtained in the previous example, the curvature tensor of the space $\mathbb{R}_{3}$ is $R_{j m n}^{i}=0$. For this reason, the geometrical objects

$$
\widetilde{\mathcal{W}}_{(3) \cdot j m n}^{i}=\delta_{m}^{i} a_{j n}-\delta_{n}^{i} a_{j m} \text { and } \widetilde{W}_{(3) \cdot j m n}^{i}=0,
$$

are the associated basic and the associated derived invariant of the Weyl type of the mapping $f$.

\section{ACKNOWLEDGEMENT}

This paper is financially supported by Serbian Ministry of Education, Science and Technological Developments, Grant No. 174012.

The author expresses his gratitude to referees for their time dedicated to revision of this paper.

\section{REFERENCES}

[1] V. Berezovski, S. Báscó, and J. Mikeš, "Almost geodesic mappings of affinely connected spaces that preserve the Riemannian curvature." Ann. Math. Inform, vol. 45, no. 0, pp. 3-10, 2015.

[2] V. Berezovski, S. Basco, and J. Mikeš, "Diffeomorphism of Affine Connected Spaces Which Preserved Riemannian and Ricci Curvature Tensors." Miskolc Math. Notes., vol. 18, no. 1, pp. 117-124, 2017.

[3] E. Einstein, "A generalization of the relativistic theory of gravitation." Ann. of. Math., vol. 45, no. 2, pp. 576-584, 1945. 
[4] E. Einstein, "Bianchi identities in the generalized theory of gravitation." Can. J. Math., no. 2, pp. 120-128, 1950.

[5] E. Einstein, Relativistic Theory of the Non-symmetric Field. New Jersey: Princeton University Press, 1984.

[6] L. P. Eisenhart, Non-Riemannian Geometry. New York: American Mathematical Society, 1927.

[7] L. P. Eisenhart, "Generalized Riemannian spaces." Proc. Natl. Acad. Sci. USA., vol. 37, pp. 311 $315,1952$.

[8] J. Mikeš, V. E. Berezovski, E. Stepanova, and H. Chudá, "Geodesic Mappings and Their Generalizations.” J. Math. Sci., vol. 217, no. 5, pp. 607-623, 2016, doi: DOI 10.1007/s10958-016-2993-9.

[9] J. Mikeš, V. Kiosak, and A. Vanžurová, Geodesic mappings of manifolds with affine connection. Olomouc: Palacky University, 2008.

[10] J. Mikeš, E. Stepanova, A. Vanžurova, and et al., Differential geometry of special mappings. Olomouc: Palacky University, 2015.

[11] J. Mikeš, A. Vanžurová, and I. Hinterleitner, Geodesic mappings and some generalizations. Olomouc: Palacky University, 2009.

[12] S. M. Minčić, "Curvature tensors of the space of non-symmetric affine connexion, obtained from the curvature pseudotensors." Mat. Vesnik, vol. 13, no. 28, pp. 421-435, 1976.

[13] S. M. Minčić, "Independent curvature tensors and pseudotensors of spaces with non-symmetric affine connexion." Coll. Math. Soc. János Bolayai, 31. Dif. geom., Budapest (Hungary), pp. 445460,1979

[14] S. M. Minčić, "On Ricci Type Identities in Manifolds With Non-Symmetric Affine Connection." Publ. Inst. Math., Nouv. Ser., vol. 94, no. 108, pp. 205-217, 2013.

[15] M. Z. Petrović and M. S. Stanković, "Special Almost Geodesic Mappings of the First Type of Non-symmetric Affine Connection Spaces.” M. S. Bull. Malays. Math. Sci. Soc., vol. 40, no. 3, pp. 1353-1362, 2017.

[16] N. S. Sinyukov, Geodesic mappings of Riemannian spaces, (in Russian). Moscow: Nauka, 1979.

[17] T. Y. Thomas, "On the projective and equi-projective geometries of paths." Proc. Nat. Acad. Sc., vol. 11, pp. 199-203, 1925.

[18] N. O. Vesić and M. S. Stanković, "Invariants of Special Second-Type Almost Geodesic Mappings of Generalized Riemannian Space.” Mediterr. J. Math., 2018, doi: https://doi.org/10.1007/s00009018-1110-3.

[19] N. O. Vesić, L. S. Velimirović, and M. S. Stanković, "Some Invariants of Equitorsion Third Type Almost Geodesic Mappings.” Mediterr. J. Math., vol. 15, no. 2, pp. 4581-4590, 2018.

[20] H. Weyl, "Zur infinitesimal geometrie: Einordnung der projectiven und der konformen auffssung." Gottingen Nachrichten, pp. 99-112, 1921.

[21] M. L. Zlatanović, "New projective tensors for equitorsion geodesic mappings." Appl. Math. Lett., vol. 25 , no. 5, pp. 890-897, 2012.

Author's address

Nenad O. Vesić

University of Niš, Department of Mathematics, Višegradska 33, 18000 Nišs, Serbia

E-mail address: n.०.vesic@out look.com 